\title{
Load Aggregator-Based Integrated Demand Response for Residential Smart Energy Hubs
}

\author{
Wenjie Lv, ${ }^{1}$ Jian Wu, ${ }^{1}$ Zhao Luo $\left(\mathbb{D},{ }^{2}\right.$ Min Ding, ${ }^{1}$ Xiang Jiang, ${ }^{3}$ Hejian Li, ${ }^{2}$ and Qian Wang \\ ${ }^{1}$ State Grid Suzhou Power Supply Company, Suzhou 215004, China \\ ${ }^{2}$ Faculty of Electric Power Engineering, Kunming University of Science and Technology, Kunming, China \\ ${ }^{3}$ Electric Power Information Technology Co., Ltd., Nanjing 210008, China
}

Correspondence should be addressed to Zhao Luo; waiting.1986@live.com

Received 8 January 2019; Revised 10 March 2019; Accepted 28 March 2019; Published 18 April 2019

Academic Editor: Alessandro Naddeo

Copyright (C) 2019 Wenjie Lv et al. This is an open access article distributed under the Creative Commons Attribution License, which permits unrestricted use, distribution, and reproduction in any medium, provided the original work is properly cited.

\begin{abstract}
In order to attract more flexible resource to take part in integrated demand response (IDR), this can be realized by introducing load aggregator-based framework. In this paper, based on residential smart energy hubs (S.E. Hubs), a two-level IDR framework is proposed, in which S.E. Hub operators play the role of load aggregators. The framework includes day-ahead bidding and real-time scheduling. In day-ahead bidding, S.E. Hub operators have to compete dispatching amount for maximal profit; hence, noncooperative game approach is formulated to describe the competition behavior among operators. In real-time scheduling, the dispatching model is formulated to minimize the error between real-time scheduling amount and bidding amount. Moreover, in order to reduce the influence of IDR on residential users, 4 categories of users' flexible loads are modeled according to load consumption characteristic, and then these models are considered as the constraints in real-time scheduling. A case study is designed to validate the effectiveness of the proposed two-level IDR framework. And simulation results confirm that smart grid, S.E. Hub operators, and residential users can benefit simultaneously.
\end{abstract}

\section{Introduction}

In recent years, energy demand in all walks is increased rapidly due to the development of economy and society. Particularly, in peak energy demand hours, tense situation of supply and demand happens from time to time, which affects the stability of smart grid. Accordingly, demand response (DR), which is one of the core technologies in smart grid, is taking an increasing, important role in digging up demand side resources and relieving tension problem of supply and demand $[1,2]$. Furthermore, with the appearance of environmental pollution and energy crisis, integrated energy system including electricity, natural gas, and heat is introduced into demand side. Consequently, traditional demand response gradually develops towards integrated demand response (IDR) $[3,4]$. The implementation of IDR project can relieve the supply pressure of electricity and promote synergetic benefits of multiply energy.

However, in general, the current DR potential lack of in-depth excavation and response degree of users is not very high. Particularly, in residential demand side, since DR resource level for single residential user is too low to participate in the DR, too much flexible resource is wasted in residential demand side. Based on such background, the concept of load aggregator (LA) gradually appears, whose role is mainly to integrate DR resource to participate in the grid dispatching [5]. Such organization is an intermediate provider, who aggregates users' flexible resource and then sells the aggregated resource to Grid Company. Under the participation of load aggregator, more flexible loads in residential demand side can take part in the DR project to obtain more profit. At the same time, power grid can realize the energy demand reduction in peak hours to guarantee the stability and safety of grid $[6,7]$.

Currently, remarkable work has been done with respect to LA. References [8-11] have proposed optimal bidding strategies of flexible loads for LA. These studies propose different approaches according to the assumptions whether the LA's bidding strategy can influence market price or whether LA's awarded electricity is exactly equal to its demand bid. 
On the other hand, in [12], a bilevel model is presented to schedule the procurement strategies, i.e., the upper level problem intends to maximize the profit of the proactive distribution company, while the lower-level one expresses the profit maximization per LA. Authors in [13] have provided an optimization algorithm to manage a virtual power plant (i.e., LA) composed of a large number of customers with thermostatically controlled appliances. Furthermore, [14] has proposed a contract-based direct control policy for the energy management of industrial thermal loads with central controller (i.e., aggregator), and then a robust model predictive control design is considered to provide upward and downward regulating power services. In [15], LA is employed to implement the direct thermostat control program for residential consumers, and for that, the paper has proposed the optimal bidding strategies and compensation mechanisms. Similarly, authors in [16] put forward a two-layer optimal dispatching model, in which LA is employed to implement the direct control of commercial users' air conditioners to reduce the electricity demand in peak hours. Authors in [17] also put forward a two-layer optimal dispatching model by LA integrating electric vehicles to participate in the DR, and simulation results show that LA can reduce the scheduling deviation and charging cost effectively.

In general, the existing research on LA is mainly focused on the scheduling of single type of appliances, such as thermal/cooling load or electric vehicle, but only a few research studies concentrate on the collaborative scheduling of different types of appliances. Moreover, few literatures have considered the IDR by aggregator scheduling different types of appliances in combined heating and power (CHP) system. Therefore, this paper mainly concentrates on the optimization of bidding strategy and control strategy for smart energy hub (S.E. Hub) by considering different categories of household appliances in CHP system. Here, S.E. Hub mainly refers to intelligent multicarrier CHP systems, which integrate CHP energy hubs and the advancement in smart grid technologies [18-20]. S.E. Hub can use energy management system (EMS) to access the electricity and natural gas prices data and wisely manage their daily energy consumption [21]. Accordingly, in our proposed framework, S.E. Hub operator is responsible for load aggregation of internal residential users, and residential users allow their different categories of flexible appliances to be controlled by S.E. Hub's EMS. In the bidding market, noncooperative game approach is proposed to describe the competition behavior among S.E. Hub operators. Furthermore, in order to guarantee the comfort of users, consumption characteristics of household appliances are modeled as the control constraints in the process of real-time scheduling. In brief, the contributions of this paper are as follows:

(1) A novel IDR framework is introduced by S.E. Hub operator aggregating different categories of flexible household appliances.

(2) A two-level dispatching architecture is formulated to reduce electricity demand in peak hours, in which day-ahead bidding amount is optimized with noncooperative game and real-time scheduling is performed based on the consumption characteristic of appliances.
(3) Simulations are conducted to validate the effectiveness and efficiency of the proposed approach via 3 S.E. Hubs participating in IDR.

The rest of this paper is organized as follows. IDR framework is introduced in Section 2. The system model is described in Section 3. And then, noncooperative game approach for day-ahead bidding is presented in Section 4 . Section 5 mainly focuses on the real-time scheduling. Case study is simulated and discussed in Section 6. Finally, Section 7 gives the conclusion.

\section{Framework Design of S.E. Hub Participating in IDR}

The structure of proposed scenario is shown in Figure 1. Each S.E. Hub can obtain continuous energy supply from public grid and gas pipeline. Energy demand of residential users is divided into electrical load and thermal load. Thermal load can be satisfied with household appliance or provided uniformly with gas turbine and gas boiler. Note that cooling load of users has been included in electrical load considering that uniformed cooling supply is not very common at present. S.E. Hub EMS can control household appliances of residential users who are willing to participate in the IDR. In addition, EMS can schedule energy output of turbine and boiler and can communicate with public grid.

In the proposed scenario, S.E. Hub operator is the intermediary between dispatching center of smart grid and IDR resource. The commercial framework of S.E. Hub operator participating in IDR is shown in Figure 2. It shows that the commercial IDR framework can be divided into macro level and micro level. Dispatching center of smart grid and S.E. Hub operators consist of macro level, while S.E. Hub operators and residential users consist of micro level. In macro level, S.E. Hub operators will have trading with dispatching center. In micro level, each S.E. Hub operator will schedule and manage flexible load of native users. The concrete steps of the framework are presented as follows:

(1) Firstly, dispatching center will broadcast information about bidding price parameters and dispatching time slots to all S.E. Hubs in the day-ahead bidding market.

(2) Secondly, in day-ahead bidding market, S.E. Hub operators will bid for load dispatching amount of each time slot to maximize the self-profit based on the broadcasted information.

(3) Thirdly, in real-time scheduling, each S.E. Hub operator will dispatch users' load according to bidding result by controlling the operation state of household appliances.

It is needed to point out that, before conducting IDR project, each S.E. Hub operator will sign a contract with residential users in the Hub. The contract content mainly includes flexible load types which are willing to be controlled by operator, compensation price which operator has to pay to users, and dispatching constraints of flexible load (such as the acceptable time slot of load, comfortable requirement of user). Furthermore, flexible load of users can be divided into 4 categories:

(1) Load I: such electrical load can be shifted and interrupted, and users are not very sensitive to the consumption 


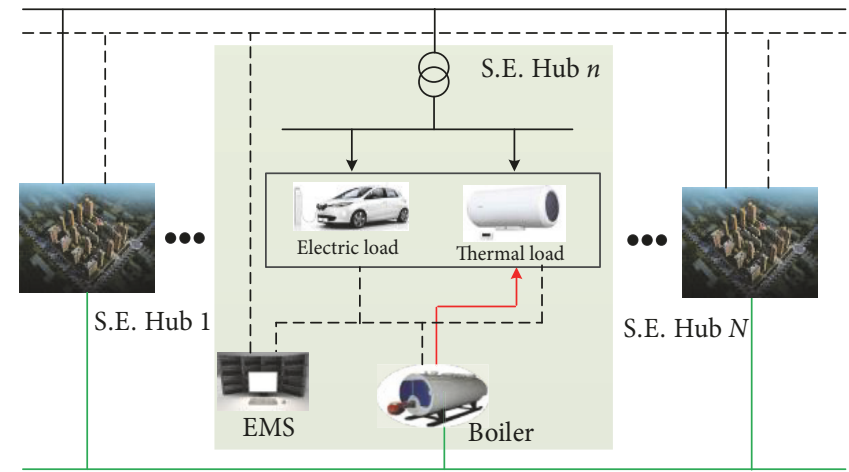

— Electricity — Gas — Heat --- Communication

Figure 1: Structure of multiple S.E. Hubs.

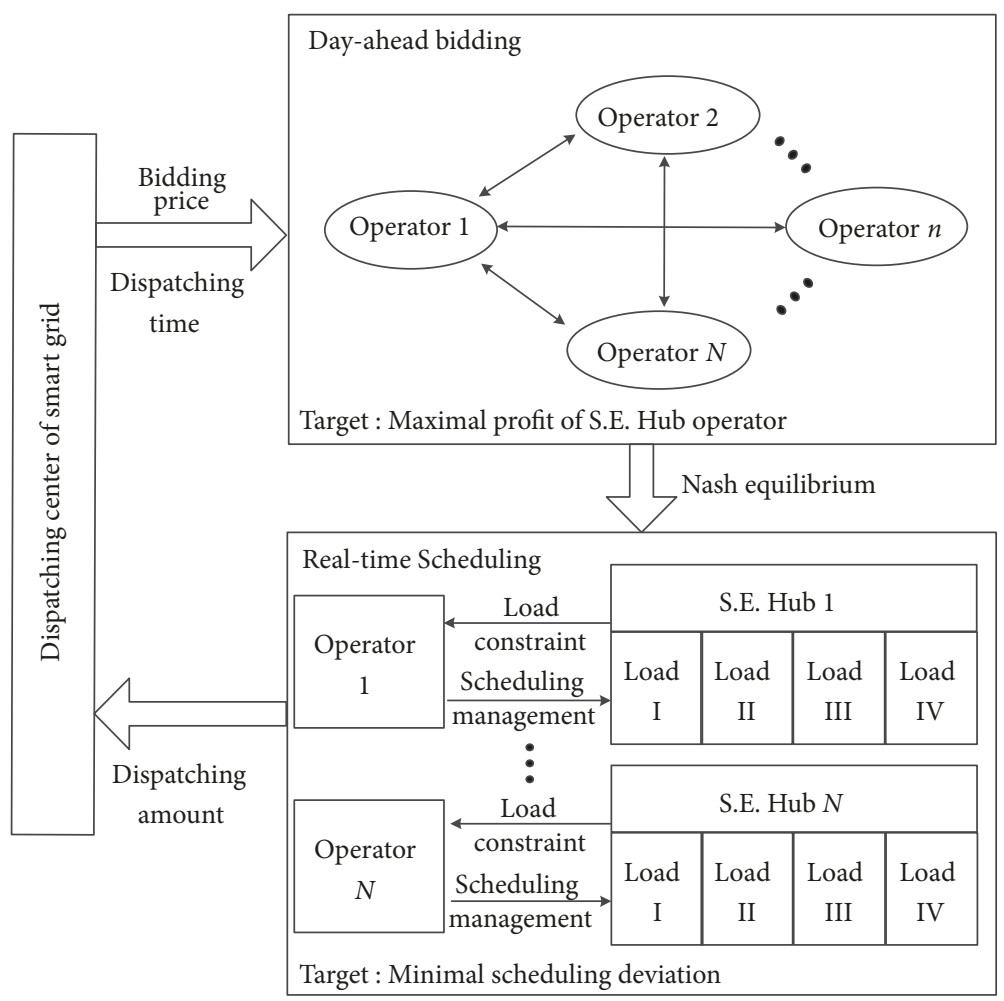

FIGURE 2: Framework for S.E. Hub participating in IDR.

time of such load. For example, users will not be influenced as long as electrical vehicle (EV) is fully charged before users go out with vehicle.

(2) Load II: such electrical load can be shifted but cannot be interrupted, and the shiftable time slot is shorter than the time slot of Load I. For example, washing machine can operate in anytime in the shiftable time slot, but it cannot be stopped until machine works over.

(3) Load III: such electrical load can be interrupted but cannot be shifted, and user comfort will be influenced if such load is interrupted inappropriately. For example, air conditioner can be stopped intermittently as long as room temperature is in the range of users' acceptable temperature.
(4) Load IV: such electrical load belongs to replaceable load and can be satisfied with electrical appliance or provided uniformly with gas turbine and gas boiler. For example, electrical water heater can satisfy users' hot water demand for bath, but the demand can also be satisfied with turbine or boiler.

After signing the contract, S.E. Hub EMS will obtain the control of above load and can control the operation state of load. According to the dispatching constraints of 4 categories of loads, S.E. Hub operator will make a reasonable control strategy to finish the dispatching amount obtained in the dayahead market. Based on the formulated framework, smart grid, S.E. Hub operators, and residential users can all obtain 
the benefit. For smart grid, load demand in peak hours can be reduced greatly, which will relieve tension problem of supply and demand. For S.E. Hub operators, they can obtain the economic profit in the trading with smart grid. For residential users, they can obtain the economic compensation from S.E. Hub operator. Additionally, since consumption characteristics of different household appliances are fully considered in real-time scheduling, users will not be influenced greatly when flexible loads are controlled by S.E. Hub operator.

\section{System Model}

Based on the proposed scenario, we assume that there are $N$ S.E. Hubs in the proposed scenario with the set $N=$ $[1,2 \ldots N]$ and load dispatching needs to be conducted in $\boldsymbol{T}=[1,2 \ldots T]$ time slots.

3.1. Energy Consumption Model. Assume that bidding amount of S.E. Hub $n$ is $L_{n}^{t}$ in time slot $t$; it is subject to the following constraint:

$$
\begin{aligned}
L_{n}^{t} & =\sum_{i=1}^{3} L_{n_{i}}^{t} \\
0 & \leq L_{n_{i}}^{t} \leq L_{n_{i}}^{\mathrm{M} t}
\end{aligned}
$$

where $L_{n_{i}}^{t}$ is the bidding amount of load $i$ in time slot $t$ and $L_{n_{i}}^{\mathrm{M} t}$ represents the maximal bidding amount of load $i$. Therefore, the individual feasible bidding amount set of S.E. Hub $n$ can be expressed as

$$
\begin{aligned}
& \mathscr{L}_{n} \\
& \quad=\left\{\boldsymbol{L}_{n}: L_{n}^{t}=\sum_{i=1}^{3} L_{n_{i}}^{t} \text { and } 0 \leq L_{n_{i}}^{t} \leq L_{n_{i}}^{\mathrm{M} t}, \forall t \in \boldsymbol{T}\right\}
\end{aligned}
$$

where $\boldsymbol{L}_{n}=\left\{L_{n}^{1}, L_{n}^{2}, \ldots, L_{n}^{T}\right\}$ represents the bidding amount profile of S.E. Hub $n$ in all dispatching slots $\boldsymbol{T}$. Accordingly, the feasible energy consumption set of all S.E. Hub operators can be expressed as

$$
\mathscr{L}=\mathscr{L}_{1} \times \mathscr{L}_{2} \cdots \times \mathscr{L}_{N}
$$

3.2. Gas Boiler Model. When residual heat in the heat recovery boiler cannot satisfy the heat demand of users, gas boiler can provide extra heat with the high efficiency by burning natural gas. Heat energy output of gas boiler can be expressed as $[22,23]$

$$
H_{n}^{t}=\eta_{b} \lambda_{\text {gas }} \gamma_{n}^{t}
$$

where $H_{n}^{t}$ is gas boiler's heat output of S.E. Hub $n$ in time slot $t$ and $\gamma_{n}^{t}$ is gas consumption rate.

3.3. Bidding Price Model. In order to maintain bidding market stability, it is necessary for dispatching center of smart grid to design a reasonable bidding price model. Assume that bidding price in time slot $t \in \boldsymbol{T}$ is $p_{t}$, and bidding amount of
S.E. Hub operator $n$ is $L_{n}^{t}$ in time slot $t$. Then, the total bidding dispatching amount in time slot $t$ is calculated as

$$
L^{t}=\sum_{n=1}^{N} L_{n}^{t}
$$

Consider that market price generally has a significant linear relation with load demand level; that is,

$$
p_{t}=\tilde{a}_{t}\left(Q^{t}-L^{t}\right)+\tilde{b}_{t}
$$

where $\widetilde{a}_{t}$ and $\widetilde{b}_{t}$ are constants and $Q_{t}$ is the predicted load amount in time slot $t$. Since predicted load amount $Q_{t}$ is irrelevant with bidding amount $L^{t}$, hence (6) can be rewritten to

$$
p_{t}=a_{t} L^{t}+b_{t} Q_{t}
$$

where $a_{t}<0$ and $b_{t}>0$ are only correlated with time $t$. Model (7) shows that $p_{t}$ is positively correlated with $Q_{t}$, while it is negatively correlated with $L^{t}$. When load level of grid is high, S.E. Hub operator will obtain a high bidding price. However, with the increase of S.E. Hub operators participating in the bidding market, the price will be reduced gradually. Therefore, the founded bidding price model can motivate the enthusiasm of S.E. Hub operators and also can guarantee the stability of market price.

\section{Noncooperative Game for S.E. Hub Participating in Day-Ahead Bidding}

According to Figure 2, S.E. Hub operator will firstly take part in the day-ahead bidding market. In the bidding market, considering each S.E. Hub operator is only concerned about self-interest, noncooperative game model is designed for operator to maximize the daily profit.

4.1. Day-Ahead Bidding Optimization Problem. The obtained profit of S.E. Hub operator is mainly from dispatching center for the contribution in peak load shifting. Considering the fact that S.E. Hub operator has to obtain the control of appliances in IDR, users will get the corresponding economic compensation. Accordingly, daily profit of S.E. Hub operator $n$ can be expressed as

$$
u_{n}=\sum_{t=1}^{T}\left(p_{t} L_{n}^{t}-\sum_{i=1}^{3} \gamma_{i} L_{n_{i}}^{t}+p_{t}^{h} L_{n_{4}}^{t}-c_{\mathrm{gas}} g_{n}^{t}\right)
$$

where $i=1-4$ represents load I-IV; $\gamma_{i}$ is the compensation price for load $i ; p_{t}^{h}$ is thermal energy price selling to users; $c_{g a s}$ is the price of natural gas; and $g_{n}^{t}$ is the gas consumption amount, which can be calculated as

$$
g_{n}^{t}=\frac{L_{n_{4}}^{t}}{\eta_{b} \lambda_{\mathrm{gas}}}
$$

In this paper, the value of $\gamma_{i}$ satisfies $\gamma_{1}<\gamma_{2}<\gamma_{3}$. Users will almost not be influenced as load I is controlled, while users will be influenced greatly as load IV is interrupted 
inappropriately. In addition, since load IV belongs to replaceable load, S.E. Hub operator would not shift its consumption time or interrupt its operation. Therefore, it is unnecessary to give corresponding economic compensation. But S.E. Hub operator can make a profit by selling heat to users.

Accordingly, S.E. Hub operator will take the maximization of daily profit (8) as the target to compete with other operators in the bidding market. That is,

$$
\begin{array}{ll}
\operatorname{maximize} & u_{n}\left(L_{n_{i}}^{t}\right) \\
\text { s.t. } & L_{n}^{t}=\sum_{i=1}^{3} L_{n_{i}}^{t} \\
& 0 \leq L_{n_{i}}^{t} \leq L_{n_{i}}^{M t}
\end{array}
$$

Each S.E. Hub operator $n \in N$ will obtain the optimal bidding strategy by solving the problem (10).

4.2. Noncooperative Game Formulation. Market bidding price $p_{t}$ is determined by all S.E. Hub operators who participate in the market. Hence, S.E. Hub operator's profit will be influenced not only by its own bidding amount $L_{n}^{t}$, but also by the bidding amount of other operators. Therefore, the day-ahead bidding process for S.E. Hub operators belongs to typical noncooperative game. Based on the objective function (10), noncooperative game model can be formulated as follows [24]:

(i) Players: all S.E. Hub operators participating in the bidding market

(ii) Strategies: bidding amount $\boldsymbol{L}_{n}$

(iii) Payoffs: the total profit of S.E. Hub $n \in N$ in all dispatching period

$$
\begin{aligned}
R_{n}\left(\boldsymbol{L}_{n}, \boldsymbol{L}_{-n}\right) & =u_{n} \\
& =\sum_{t=1}^{T}\left[p_{t} L_{n}^{t}-\sum_{i=1}^{4} \gamma_{i} L_{n_{i}}^{t}+p_{t}^{h} L_{n_{4}}^{t}-c_{\mathrm{gas}} g_{n}^{t}\right]
\end{aligned}
$$

where $\boldsymbol{L}_{-n}=\left[\boldsymbol{L}_{1}, \ldots, \boldsymbol{L}_{n-1}, \boldsymbol{L}_{n+1}, \ldots, \boldsymbol{L}_{N}\right]$ represents the bidding strategy set of other $N-1$ S.E. Hub operators except $n$.

All S.E. Hub operators will constantly update their own strategies according to the payoff function (11) until the payoff reaches the maximum value. Once all operators obtain the maximum profit, anyone will not change the strategy. Such equilibrium state is called Nash equilibrium, which can be expressed as

$$
R_{n}\left(\boldsymbol{L}_{n}^{*}, \boldsymbol{L}_{-n}^{*}\right) \geq R_{n}\left(\boldsymbol{L}_{n}, \boldsymbol{L}_{-n}^{*}\right) \quad \forall n \in N
$$

where $\left(\boldsymbol{L}_{n}^{*}, \boldsymbol{L}_{-n}^{*}\right)$ is Nash equilibrium.

4.3. Nash Equilibrium. In this section, the existence of Nash equilibrium for the formulated noncooperative game will be proved. And then, the algorithm process for searching the equilibrium will be presented.
Lemma 1. For each S.E. Hub operator $n \in \boldsymbol{N}$, the set $\boldsymbol{L}_{n}$ is convex and compact, and function $R_{n}\left(\boldsymbol{L}_{n}, \boldsymbol{L}_{-n}\right)$ is continuously differentiable in $\boldsymbol{L}_{n}$. For the fixed tuple $\boldsymbol{L}_{-n}$, the function is concave in over the set $\boldsymbol{L}_{n}$

Proof. See Appendix A.

Definition 2. The variational inequality denoted by $\operatorname{VI}(\mathscr{L}, \boldsymbol{F})$ is to find a vector $\boldsymbol{x}^{*} \in \mathscr{L}$ such that

$$
\left(\boldsymbol{x}-\boldsymbol{x}^{*}\right)^{T} \boldsymbol{F}\left(\boldsymbol{x}^{*}\right) \leq 0 \quad \forall \boldsymbol{x} \in \mathscr{L}
$$

Based on Lemma 1 and Definition 2, the following lemma can be obtained.

Lemma 3. The Nash equilibrium of the above formulated game is equivalent to the solution of the variational inequality (VI) problem denoted by $\mathrm{VI}(\mathscr{L}, \boldsymbol{F})$, where

$$
\boldsymbol{F}(\boldsymbol{L})=\left[\boldsymbol{F}_{n}\left(\boldsymbol{L}_{n}, \boldsymbol{L}_{-n}\right)\right]_{n=1}^{N}
$$

where $\boldsymbol{L}=\left(\boldsymbol{L}_{n}, \boldsymbol{L}_{-n}\right)$ and $\boldsymbol{F}_{n}\left(\boldsymbol{L}_{n}, \boldsymbol{L}_{-n}\right)$ is expressed as follows:

$$
\boldsymbol{F}_{n}\left(\boldsymbol{L}_{n}, \boldsymbol{L}_{-n}\right)=\nabla_{\boldsymbol{L}_{n}} R_{n}\left(\boldsymbol{L}_{n}, \boldsymbol{L}_{-n}\right)
$$

Proof. The proof can be found in [25].

According to Lemma 3, we can obtain the following proposition.

Proposition 4. In the above noncooperative game, there exists a Nash equilibrium and the equilibrium is unique.

Proof. See Appendix B.

In order to search the Nash equilibrium, the algorithm process is proposed as follows.

Step 1. Initialize the parameters in the game.

Step 2. In the individual feasible bidding amount set $\mathscr{L}$, randomly initialize all S.E. Hub operators' bidding strategy $\boldsymbol{L}_{n}$.

Step 3. Let the strategy $\boldsymbol{L}_{-n}$ of other $N-1$ operators fixed; then for S.E. Hub operator $n$, maximize the function (11) to search the optimal strategy $\boldsymbol{L}_{n}$ by using interior point method, and then let $R_{n}\left(\boldsymbol{L}_{n}^{*}, \boldsymbol{L}_{-n}^{*}\right)=R_{n}\left(\boldsymbol{L}_{n}, \boldsymbol{L}_{-n}^{*}\right), \boldsymbol{L}_{n}^{*}=\boldsymbol{L}_{n}$.

Step 4. Similar to Step 3, search the optimal strategy $\boldsymbol{L}_{-n}$ of other $N$-1 S.E. Hub operators in turn and update $\boldsymbol{L}_{-n}^{*}=\boldsymbol{L}_{-n}$.

Step 5. Repeat Steps 3 and 4 until $\left(\boldsymbol{L}_{n}^{*}, \boldsymbol{L}_{-n}^{*}\right)$ is unchanged. Then, $\left(\boldsymbol{L}_{n}^{*}, \boldsymbol{L}_{-n}^{*}\right)$ is the Nash equilibrium, and the value of $R_{n}\left(\boldsymbol{L}_{n}^{*}, \boldsymbol{L}_{-n}^{*}\right)$ is the maximal profit for $\forall n \in N$.

\section{Optimization Model for S.E. Hub Participating in Real-Time Scheduling}

In real-time scheduling, each S.E. Hub operator will dispatch users' load according to day-ahead bidding result by controlling the operation state of household appliances. Accordingly, the optimization model is designed for S.E. Hub operator to search the optimal real-time scheduling strategy. 
5.1. Real-Time Scheduling Optimization Problem. After signing the contract with users, S.E. Hub operator will obtain the control of the above referred 4 categories of load. In order to reduce the influence of peak shaving on users, S.E. Hub operator has to schedule appliances according to the load consumption characteristic. Assume that there are $\boldsymbol{M}=$ $[1,2, \cdots, M]$ residential users who are willing to sign the contract with operator $n$ in the Hub. Suppose that $\mathscr{A}_{m}^{i}(i=$ $1,2,3,4)$ is the load $i$ 's set of user $m$ and $a_{m}^{i} \in \mathscr{A}_{m}^{i}$ is any appliance in the set $\mathscr{A}_{m}^{i}$. Considering that S.E. Hub operator implements dispatching mainly by controlling appliances being on or off state, assume that $S a_{m}^{i}(t)$ is the operation state of appliance $a_{m}^{i}$ in time slot $t . S a_{m}^{i}(t)=1$ represents $a_{m}^{i}$ being in off state and $S a_{m}^{i}(t)=0$ represents $a_{m}^{i}$ being on operation. Based on the above assumption, dispatching amount of S.E. Hub $n$ in real time can be expressed as

$$
\widetilde{L}_{n}^{t}=\sum_{m=1}^{M} \sum_{i=1}^{t} \sum_{a_{m}^{i} \in \mathscr{A}_{m}^{i}} S a_{m}^{i}(t) P_{t}^{a_{m}^{i}} \Delta t
$$

where $P_{t}^{a_{m}^{i}}$ is the consumption power of appliance $a_{m}^{i}$ and $\triangle t$ is the time interval.

Generally, S.E. Hub operator will take the bidding amount as the objective to schedule users' appliances in real time. However, each kind of appliance has its unique consumption constraint and control variables in real-time belong to discrete variables. Therefore, day-ahead bidding amount $L_{n}^{t}$ and real-time dispatching amount $\widetilde{L}_{n}^{t}$ are hard to be always equal. In order to maximize the profit, S.E. Hub operator will intend to minimize the deviation between $L_{n}^{t}$ and $\widetilde{L}_{n}^{t}$ via controlling the operation state of appliances. Consequently, S.E. Hub operator will take the deviation as the optimal target to control users' appliances. Accordingly, real-time scheduling model can be expressed as

$$
\underset{S a_{m}^{i}(t), t \in \boldsymbol{T}}{\operatorname{minimize}}\left(L_{n}^{t}-\widetilde{L}_{n}^{t}\right)^{2}
$$

5.2. Optimization Constraints for Real-Time Scheduling. In order to reduce the influence of IDR on users, real-time scheduling has to obey the consumption characteristic of household appliances. Therefore, the referred 4 categories of load have the following dispatching constraints.

(1) Load I: assume that the working period of such load is $\boldsymbol{T}_{1}=\left[1,2, \cdots, T_{1}\right]$; then load I has to consume the required energy in period $\boldsymbol{T}_{1}$; that is,

$$
\sum_{t \in \boldsymbol{T}_{1}}\left(1-S a_{m}^{1}(t)\right) P_{t}^{a_{m}^{1}} \Delta t=Q_{a_{m}^{1}}
$$

where $Q_{a_{m}^{1}}$ is the daily energy consumption of appliance $a_{m}^{1}$ of user $m$ and $P_{t}^{a_{m}^{1}}$ is the consumption power of appliance $a_{m}^{1}$.

(2) Load II: assume that the working period of such load is $\boldsymbol{T}_{2}=\left[1,2, \cdots, T_{2}\right]$ and $\boldsymbol{T} \subsetneq \boldsymbol{T}_{2} \subsetneq \boldsymbol{T}_{1}$; then load II has to consume the required energy in period $\boldsymbol{T}_{2}$. Furthermore, once such load starts to operate, it cannot be interrupted:

$$
\begin{array}{cl} 
& (j-k+1) P_{t}^{a_{m}^{2}} \Delta t=Q_{a_{m}^{2}} \\
\text { s.t. } & \left\{\begin{array}{l}
j=\max \left(\arg \left(S a_{m}^{2}(t)=0\right)\right) \\
k=\min \left(\arg \left(S a_{m}^{2}(t)=0\right)\right)
\end{array} \quad\left(t \in \boldsymbol{T}_{2}\right)\right.
\end{array}
$$

where $Q_{a_{m}^{2}}$ is the daily energy consumption of appliance $a_{m}^{2}$ of user $m$ and $P_{t}^{a_{m}^{2}}$ is the consumption power of appliance $a_{m}^{2}$.

(3) Load III: such load cannot be shifted from the peak hours. Hence, such load can only be interrupted intermittently in dispatching time $\boldsymbol{T}$ to reduce the consumption in peak hours. Since user's comfort will be influenced if such load is interrupted inappropriately, therefore, S.E. Hub operator has to control turn-off time and turn-on time appropriately. That is,

$$
\begin{array}{ll}
\min & (k) \Delta t \geq \tau_{\text {on }}^{a_{m}^{3}} \\
\text { s.t. } & \left\{\begin{array}{l}
S a_{m}^{3}(t-1)=1 \\
S a_{m}^{3}(t)=0 \\
S a_{m}^{3}(t+k)=1
\end{array} \quad(t \in T)\right. \\
\min \quad(j) \Delta t \leq \tau_{\text {off }}^{a_{m}^{3}} \\
\text { s.t. } \quad\left\{\begin{array}{l}
S a_{m}^{3}(t-1)=0 \\
S a_{m}^{3}(t)=1 \\
S a_{m}^{3}(t+j)=0
\end{array} \quad(t \in T)\right.
\end{array}
$$

where $\tau_{\mathrm{on}}^{a_{m}^{3}}$ is the shortest turn-on time of appliance $a_{m}^{3}$ of user $m$ and $\tau_{\text {off }}^{a_{m}^{3}}$ is the longest turn-off time of appliance $a_{m}^{3}$. The values of $\tau_{\text {on }}^{a_{m}^{3}}$ and $\tau_{\text {off }}^{a_{m}^{3}}$ are determined by user's comfort. When turn-on time is shorter than $\tau_{\text {on }}^{a_{m}^{3}}$ or turn-off time is longer than $\tau_{\text {off }}^{a_{m}^{3}}$, user's comfort will both be influenced.

(4) Load IV: such electrical load belongs to replaceable load. Hence, as long as S.E. Hub operator can provide the equal energy produced by appliance $a_{m}^{4}$, the comfort of users will not be influenced. That is,

$$
\begin{array}{ll} 
& \left(1-S a_{m}^{4}(t)\right) P_{t}^{a_{m}^{4}} \Delta t+S u_{m}(t) P_{t}^{S u_{m}} \Delta t=Q_{a_{m}^{4}} \\
\text { s.t. } \quad & S u_{m}(t)\left(1-S a_{m}^{4}(t)\right)=0 \quad(t \in \boldsymbol{T})
\end{array}
$$

where $Q_{a_{m}^{4}}$ is the energy demand of user $m$ with appliance $a_{m}^{4}$ in time slot $t ; P_{t}^{a_{m}^{4}}$ is energy output of appliance $a_{m}^{4}$ in time slot $t ; P_{t}^{S u_{m}}$ is provided energy of substitute device for users in time slot $t ; S u_{m}(t)=1$ represents substitute device providing energy for user $m$ and else $S u_{m}(t)=0$. As long as energy demand of users is fully satisfied, users are not concerned about who provides energy.

Based on the constraints (18) -(22), each S.E. Hub operator will take the model (17) as the objective to schedule appliances in dispatching time slot $\mathbf{T}$. 


\section{Case Study}

Assume that there are $N=3$ S.E. Hubs and each S.E. Hub has 1000 users. In addition, S.E. Hub operator takes charge of the daily operation of Hub and users can obtain the thermal energy uniformly from gas boiler equipped by S.E. Hub. Dispatching center of smart grid will broadcast the dispatching time slots and bidding price to all S.E. Hubs according to the predicted load in day-ahead. Considering that peak hours generally appear in 10:00-14:00 and 18:0021:00, the scheduling cycle can be divided into day scheduling and night scheduling. Here, the night scheduling (i.e., 18:0024:00) is taken as an example to show the effectiveness of the proposed approach. Accordingly, suppose that peak shaving hours are 18:00-21:00 and scheduling interval is $\Delta t=15$ minutes. That is, S.E. Hub operator will bid for load shaving amount with other 2 operators in time slots $\boldsymbol{T}=[1,2, \cdots, 12]$. Furthermore, bidding price parameters are shown as follows (unit: dollars/kWh): $a_{t}=-0.036, b_{t}=0.021$ ( $t=1 \sim 5$ and $11 \sim 12) ; a_{t}=-0.035, b_{t}=0.022(t=6 \sim 10)$. The thermal energy price $p_{t}^{h}$ is 0.025 dollars $/ \mathrm{kWh}$, natural gas price $c_{\text {gas }}$ is 0.397 dollars $/ \mathrm{m}^{3}$, and calorific value of natural gas $\lambda_{\text {gas }}$ is $9.7 \mathrm{kWh} / \mathrm{m}^{3}$. The load categories that users are willing to participate in IDR are shown in Table 1.

The concrete parameters of each household appliance are introduced as follows.

(1) Assume that the charging time slots of EVs are 18:0024:00 and 0:00-6:00. That is, the working period of EV is $\boldsymbol{T}_{1}=[1,2, \cdots, 48]$. EV's daily energy consumption is determined by energy amount for traveling $1 \mathrm{~km}$ and daily mileage. Presently, a common EV generally consumes energy $0.1-0.2 \mathrm{kWh}$ for traveling $1 \mathrm{~km}$. In addition, considering that development scale of EVs is small currently, there is a lack of relevant data about EV's daily mileage. Thus, traditional vehicles are applied to analyze driving characteristics of EVs. According to the data of American National Household Travel Survey, vehicle's daily mileage $d$ is

$$
f(d)=\frac{1}{d \sigma \sqrt{2 \pi}} \exp \left(-\frac{(\ln d-\mu)^{2}}{2 \sigma^{2}}\right)
$$

where $d$ is EV's daily mileage and $\mu=3.7$ and $\sigma=0.9$.

(2) According to market research, energy power of washing machine and dishwasher are generally about $0.5 \mathrm{~kW}$ and $0.8 \mathrm{~kW}$. In this case study, washing machine's power obeys uniform distribution on $[0.4,0.6] \mathrm{kW}$, and dishwasher's power obeys uniform distribution on $[0.6,1] \mathrm{kW}$. In addition, assume that working time slots of washing machine and dishwasher are 18:00-24:00; that is, $\boldsymbol{T}_{2}=[1,2, \cdots, 20]$, and single-use time cycle is half an hour.

(3) Air conditioner can be employed to generate cooling energy in summer or thermal energy in winter. Therefore, air conditioner in summer can be considered as load III, which can be interrupted intermittently, while air conditioner in winter can be considered as load VI, which can be substituted by gas boiler. Assume that working time slots of air conditioner are 18:00-24:00 and 0:00-6:00, and scheduling time slots are 18:00-21:00. When air conditioner is used in winter, assume that thermal power of conditioner obeys uniform distribution on $[1.8,2.2] \mathrm{kW}$ and $\eta=3.34$ is energy efficiency ratio of air conditioner. Therefore, thermal power that S.E. Hub has to provide with gas boiler is about 6.0 7.3 $\mathrm{kW}$ in time slot $\boldsymbol{T}$. When air conditioner is used in summer, dynamic model can be expressed as

$$
\begin{aligned}
& T_{\max }=T_{\text {out }}\left(1-\varepsilon^{\tau_{\text {off }}}\right)+T_{\min } \varepsilon^{\tau_{\text {off }}} \\
& T_{\text {min }}=\left(T_{\text {out }}-\frac{\eta \bar{P}}{A}\right)\left(1-\varepsilon^{\tau_{\text {on }}}\right)+T_{\text {max }} \varepsilon^{\tau_{\text {on }}}
\end{aligned}
$$

where $\bar{P}=1.2$ is average energy consumption power of air conditioner in summer; $T_{\text {out }}=33^{\circ} \mathrm{C}$ is the outside temperature; $\left[T_{\min }, T_{\max }\right]=\left[24^{\circ} \mathrm{C}, 27^{\circ} \mathrm{C}\right]$ is room temperature interval which residential users can accept; $\varepsilon=0.96$ is coefficient of heat dissipation; and $A=0.18$ is thermal conductivity. After some calculation, $\tau_{\text {on }}=5$ minutes; $\tau_{\text {off }}=10$ minutes. Accordingly, control time interval of air conditioner in summer is 5 minutes.

(4) Assume that the users have the demand of hot water for bath in time slot 18:00-23:00. According to market research, energy power of electric heater is generally about $2 \mathrm{~kW}$. In this case study, heater's power obeys uniform distribution on $[1.8,2.2] \mathrm{kW}$. Assume that the number of users for bath in 18:00-21:00 takes 60\% in all users, and singleuse time cycle is 15 minutes.

6.1. Dispatching Result in Summer. In order to reduce the control difficulty of S.E. Hub operator, we assume that 1000 users in each S.E. Hub are equally divided into 10 groups. Furthermore, considering that it is hard to persuade all users to sign the contract, we assume that S.E. Hub 1 has $80 \%$ participation rate, S.E. Hub 2 has $90 \%$ participation rate, and S.E. Hub 3 has $100 \%$ participation rate. That is, S.E. Hub operator 1-3 can control 8-10 groups of users' appliances, respectively. Operator will take each group as the control unit; that is, operation state of appliances in the same group will be controlled uniformly.

According to the above assumption, optimal result in bidding market and real-time scheduling can be obtained. Figure 3 is the bidding result of 3 S.E. Hub operators in the market. From the figure, we can see that the bidding amount is increased gradually from 18:00 to 21:00, especially during 19:15-20:30, and S.E. Hub operator with more flexible DR resources will bid for more dispatching amount. The reason is that the 4 types of appliances are gradually put into operation from 18:00 to 21:00; hence, S.E. Hub operator can control more appliances, while at 19:15-20:30, since such time slots have a high load level with high bidding price, each S.E. Hub operator will compete the maximal bidding amount based on their own flexible resources. From the aspect of smart grid, by introducing market price into the bidding mechanism, S.E. Hub operators can be encouraged into the noncooperative game. Consequently, the dispatching amount will be determined according to load level of power system and can also reduce dispatching center's IDR cost.

According to the bidding amount in the day-ahead market, the actual reduction amount of 3 S.E. Hub operators is shown in Table 2. From the table, we can see that each 
TABLE 1: Load categories participating in DR in summer and winter.

\begin{tabular}{lcccc}
\hline Season & Load I & Load II & Load III & Load IV \\
\hline Summer & EV & Dishwasher Washing machine & Air conditioner & Heater \\
Winter & EV & Dishwasher & None & Air conditioner \\
\hline$\gamma_{i}$ & $\gamma_{1}=0.022$ & $\gamma_{2}=0.035$ & $\gamma_{3}=0.061$ & $\gamma_{4}=0$ \\
\hline
\end{tabular}

TABLE 2: Actual reduction amount of 3 S.E. Hub operators in summer (MWh).

\begin{tabular}{lccccc}
\hline Operator & EV & Dishwasher & Washing machine & Air conditioner & Electric heater \\
\hline 1 & 1.60 & 0.26 & 0.16 & 1.51 & 0.66 \\
2 & 1.76 & 0.29 & 0.18 & 1.48 & 0.70 \\
3 & 2.04 & 0.31 & 0.2 & 1.46 & 0.82 \\
\hline
\end{tabular}

TABLE 3: Actual reduction amount of 3 S.E. Hub operators in winter (MWh).

\begin{tabular}{llcr}
\hline Operator & EV & Dishwasher & Air conditioner \\
\hline 1 & 1.58 & 0.26 & 2.18 \\
2 & 1.76 & 0.25 & 2.42 \\
3 & 2.06 & 0.22 & 2.71 \\
\hline
\end{tabular}

household appliance has reduced the energy consumption during peak hours, but EVs and air conditioners have the greatest contribution. It shows that EVs and air conditioners play an important role in the DR. Here, it is needed to state that energy consumption of EV is reduced by shifting demand into other time slots, while air conditioner's consumption is reduced by interrupting air conditioner. Furthermore, in order to show the control strategy of each appliance, the control result of group 1 is shown in Figure 4, in which, Figures 4(a) and 4(b) represent the operation state of EVs, dishwasher and washing machine, electric heat, and air conditioner, respectively. From the figure, we can see the real-time control strategy of S.E. Hub operator. EVs are charged between $3: 15$ and 5:15. Operation times of dishwashers and washing machine have been shifted into 22:00-22:30 and 23:00-23:30, respectively. Users' demands of hot water are all provided by gas boiler in 18:00-21:00, and hot water will be provided by electric load if users have demand after 21:00. While for air conditioner, S.E. Hub operator will interrupt the conditioner intermittently during 18:00-21:00 and then air conditioner will not be controlled after 21:00. Due to space limitation of paper, the control strategies of other groups are not presented in the paper. After all S.E. Hub operators schedule energy consumption, a new load profile can be obtained, which is shown in Figure 5. By comparing the original profile and scheduling profile, energy demand in peak hours from 18:00 to 21:00 has greatly reduced and energy demand in off-peak hours has also increased to a certain degree. It demonstrates that the proposed IDR mechanism has a good performance in peak shaving and valley filling. In order to analyze the performance quantitatively, peak-to-average ratio (PAR) is introduced to measure the fluctuating level of load [22]. After some calculation, PAR in original profile is 2.13 , while PAR in scheduling profile is 1.74 . The reduction of PAR has reduced to $18.3 \%$. It shows that fluctuating level of load has been weakened greatly after implementing IDR.

6.2. Dispatching Result in Winter. Similarly, we still assume that S.E. Hub operators 1-3 have the control of 8-10 groups of users' appliances, respectively. Figure 6 shows the bidding amount of 3 S.E. Hub operators in winter. It shows that 3 operators all obtain the highest bidding amount during 19:1520:30 due to the high load level with high bidding price in these time slots. Therefore, S.E. Hub operators will have a higher profit for selling more resource in these time slots compared with other times. Additionally, by comparing result in Figure 3, we can find that during 18:00-19:15 and 20:3021:00, the range of bidding amount of 3 S.E. Hub operators in winter has reached $[49,87] \mathrm{kWh}$. The main reason is that thermal demand of users in winter will increase greatly and such demand can also be satisfied by gas boiler; hence, S.E. Hub with more users will have more flexible DR resources. Furthermore, it is unnecessary for S.E. Hub operator to pay the economic compensation to users when thermal demand of users is substituted with gas boiler. Consequently, S.E. Hub operator with more thermal DR resources will bid for more dispatching amount in the market.

Table 3 is the actual reduction amount of 3 S.E. Hub operators in winter. From the table, one can see that substituting air conditioner with gas boiler will greatly improve the effect of S.E. Hub participating in IDR. Since economic compensation of dishwasher in 3 appliances is the highest, actual reduction amount of dishwasher will be less in S.E. Hub with more IDR resources. For example, energy demand of dishwasher in S.E. Hub 1 is reduced to $0.26 \mathrm{MWh}$, while it is reduced to $0.22 \mathrm{MWh}$ in S.E. Hub operator 3. According to the scheduling result, we can obtain the load profile in winter, which is shown in Figure 7. One can see that the effect of 
TABLE 4: Deviation between actual reduction amount and bidding amount (kWh).

\begin{tabular}{lcccc}
\hline Season & Time slots & Operator 1 & Operator 2 & Operator 3 \\
\hline \multirow{4}{*}{ Summer } & 6 & 1.21 & 0.82 & 0 \\
& 7 & 0.96 & 0 & 0 \\
& 8 & -0.06 & -0.92 & 0 \\
& 9 & 0 & 1.07 & 0.31 \\
Winter & 10 & 2.12 & 0.11 & 0 \\
& 6 & 0.81 & 0 & 0.71 \\
& 7 & 0 & -0.61 & 0 \\
\end{tabular}

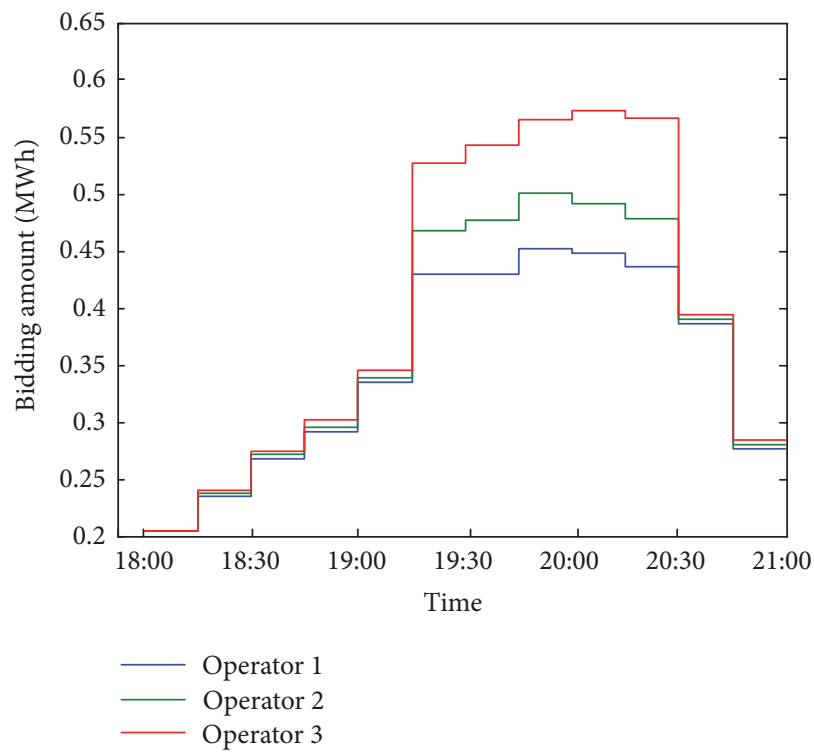

FIgURE 3: Bidding amount of 3 S.E. Hub operators in summer.

peak shaving in 18:00-21:00 is weaker than that in summer, mainly because flexible loads in winter are less than loads in summer. After some calculation, PAR in original profile is 2.09, while PAR in scheduling profile is 1.78 . The reduction of PAR has reduced to $14.5 \%$, which is slightly weaker than that in summer.

6.3. Control Deviation and Profit Result. In this section, control deviation and profit of each S.E. Hub operator will be discussed and analyzed. Table 4 shows the deviation between actual reduction amount and bidding amount. Comparing the order of magnitude of bidding amount (i.e., MWh) and deviation (i.e., $\mathrm{kWh}$ ), it is obvious that deviation is very small. Furthermore, from the prospect of deviation in summer and winter, we can find that deviation in summer is generally more than deviation in winter in most of time slots. It is mainly because that in summer, air conditioners' operation state in a group is uniformly controlled as a unit; hence, the frequent switching of air conditioners increases the probability of large deviation. Here, it is needed to classify that when a group contains less than 1000 users (e.g., 500 users or 100 users), the deviation will be reduced, but the controlling difficulty for S.E. Hub operator will increase. Table 5 shows the economic compensation and profit situation of 3 S.E. Hub operators. From the table, one can see that S.E. Hub operator with more flexible resources will obtain more overall profit. From the prospect of different seasons, S.E. Hub operators obtain more profit in winter than that in summer. In addition, operators in summer have to pay for more economic compensation than that in winter. The reason is that air conditioner in winter belongs to load IV; hence, operators don't need to make up for residential users. Furthermore, S.E. Hub operator can obtain more thermal profit in winter because of large thermal demand of users.

6.4. Analysis of Prediction Accuracy. In the above analysis, there is a potential assumption. That is, S.E. Hub operator can predict IDR resource amount precisely in day-ahead. However, in the reality, it is impossible that day-ahead prediction accuracy can reach $100 \%$ due to the randomness 
TABLE 5: Profit analysis of 3 S.E. Hub operators (dollars).

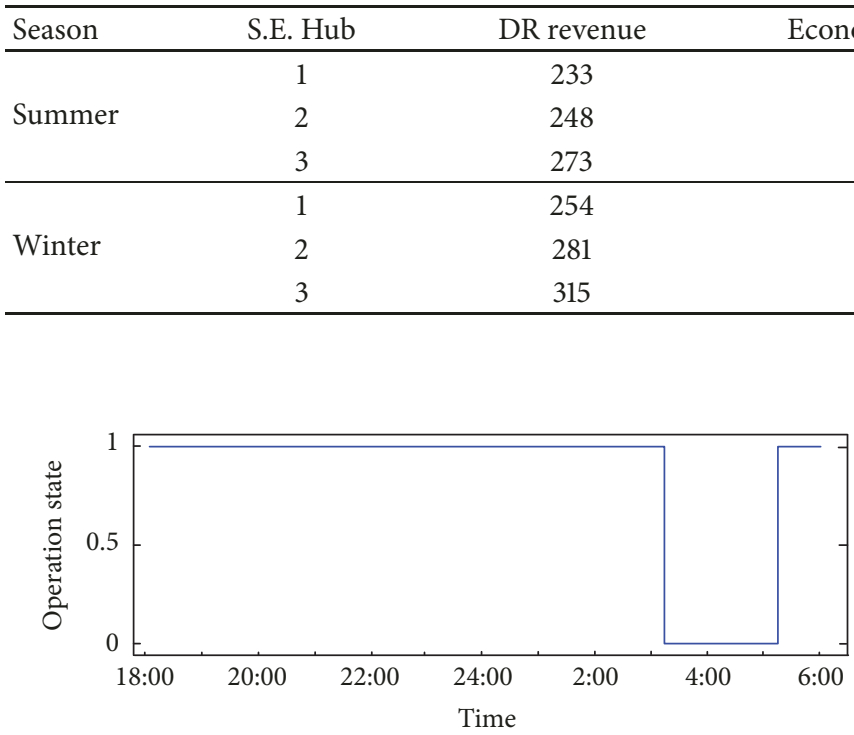

(a)

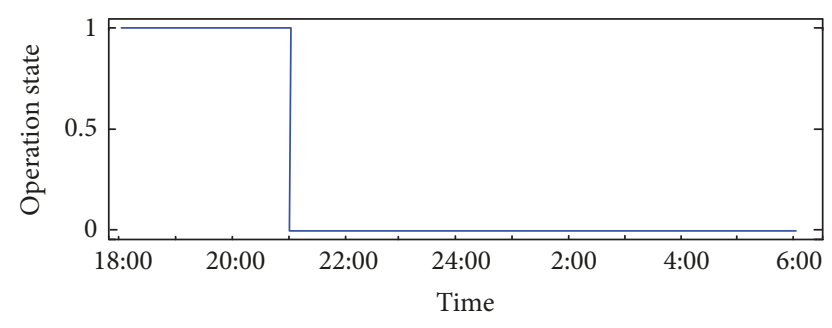

(c)

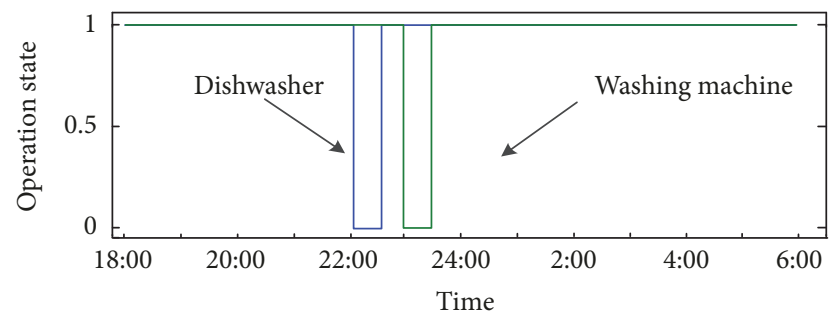

(b)

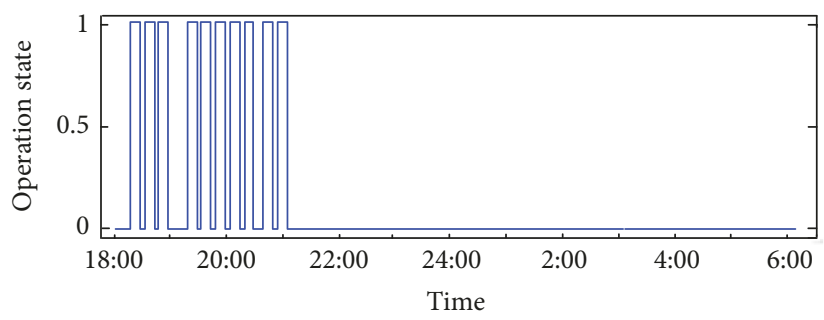

(d)

FIGURE 4: Control strategy of group 1.

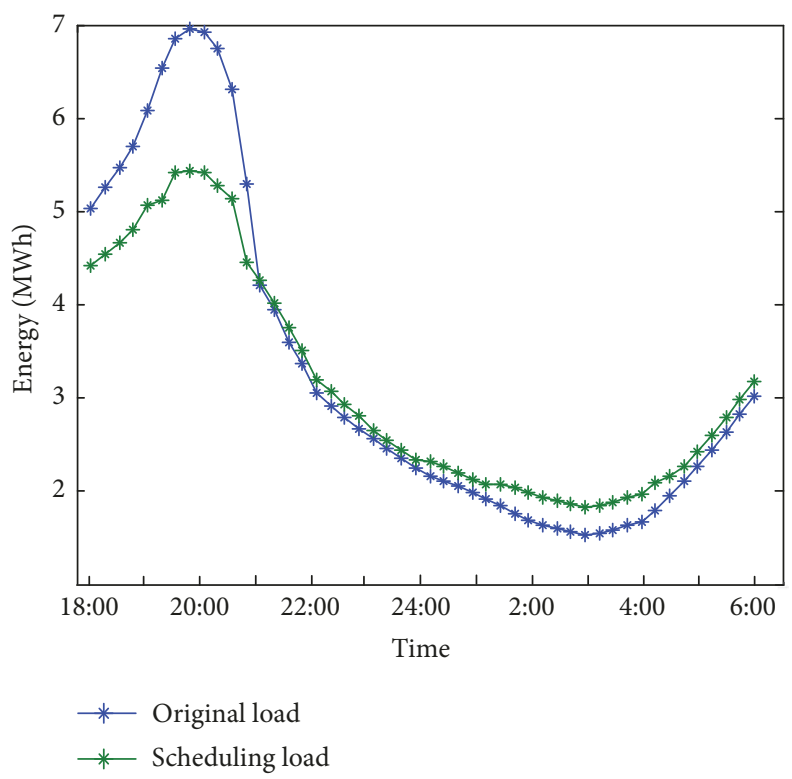

FIGURE 5: Load profile of public grid in summer. 


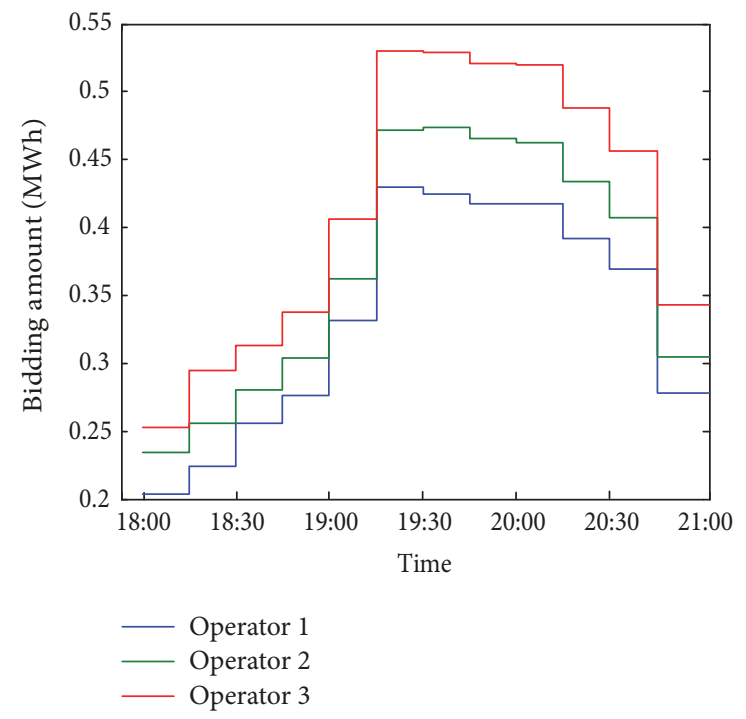

FIGURE 6: Bidding amount of 3 S.E. Hub operators in winter.

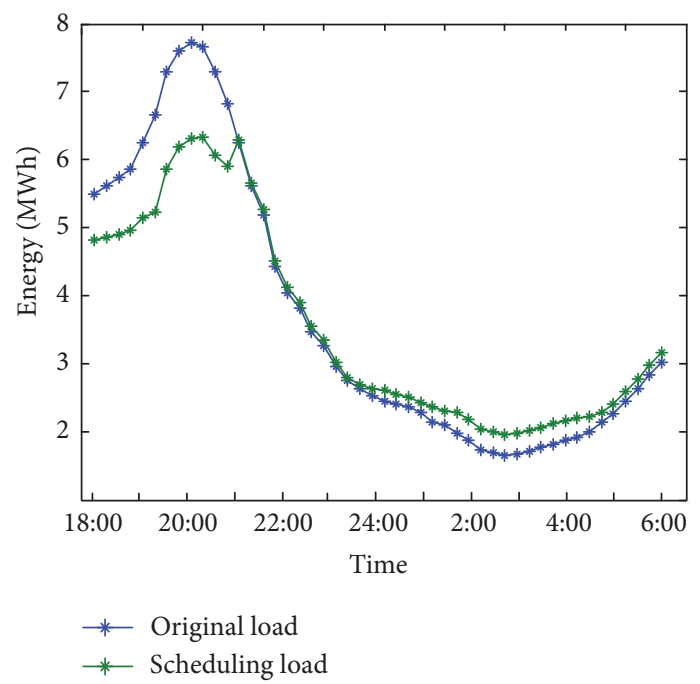

FIGURE 7: Load profile of public grid in winter.

of users' consumption. The prediction accuracy has a great influence on the dispatching result. In this section, we assume that forecasting result of IDR resource amount has a $0 \%$ $20 \%$ deviation compared with the real amount, and then the dispatching and profit result will be analyzed. Here, $0 \%-20 \%$ deviation mainly refers to the situation that real IDR resource amount is less than the forecasting amount.

Figures 8 and 9 are the profit and scheduling deviation under different prediction accuracy in summer and winter, respectively. In the figure, scheduling deviation is the summation of 3 operators' deviation in total time slots and profit is the summation of $3 \mathrm{~S}$.E. Hub operators. One can see that, with the increase of prediction deviation, the scheduling deviation has increased from 0.007 MWh to 1.763 MWh in summer and from 0.002 MWh to $1.763 \mathrm{MWh}$ in winter, while profit has decreased from 347 dollars to 297 dollars in summer and from 822 dollars to 749 dollars in winter. Particularly, when prediction accuracy varies in $0 \%$ $12 \%$, prediction accuracy has little influence on real-time scheduling deviation and profit. However, when prediction accuracy varies in 16\%-20\%, prediction accuracy will greatly affect real-time scheduling deviation and profit. The main reason is that day-ahead bidding amount is less than realtime flexible load amount when prediction accuracy varies in $0 \%-12 \%$; hence, S.E. Hub operators can achieve the bidding amount by controlling more household appliances. But when bidding amount exceeds real-time flexible load amount, it is impossible for S.E. Hub operators to achieve the bidding amount. Consequently, the real-time scheduling deviation will increase rapidly and profit will also decrease dramatically. Based on the above analysis, the prediction accuracy has a great influence on the scheduling result and profit of S.E. Hub operator. Therefore, it is important and necessary for S.E. Hub operator to improve the prediction accuracy of IDR resource. 


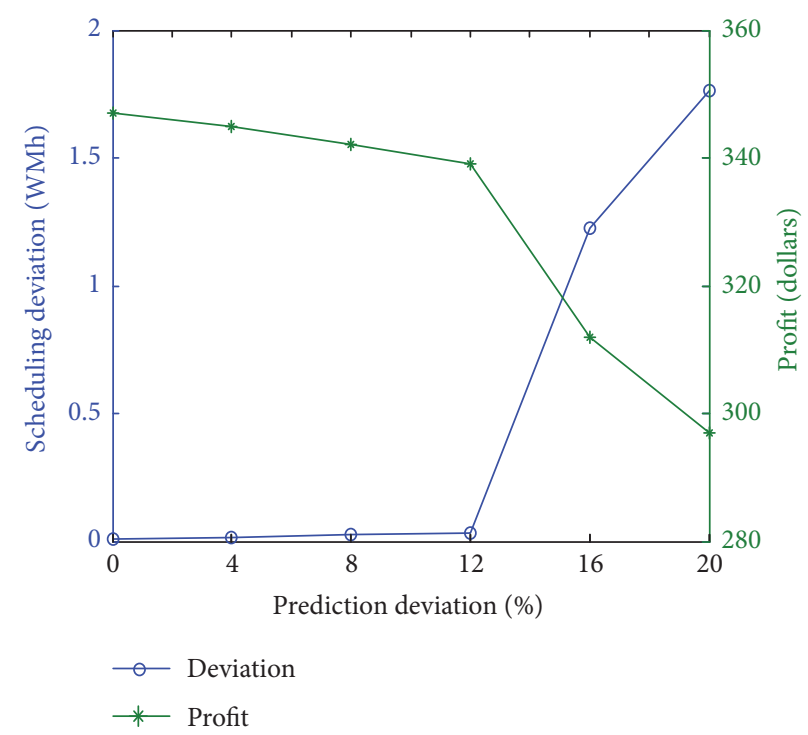

FIgUre 8: Profit and scheduling deviation in summer.

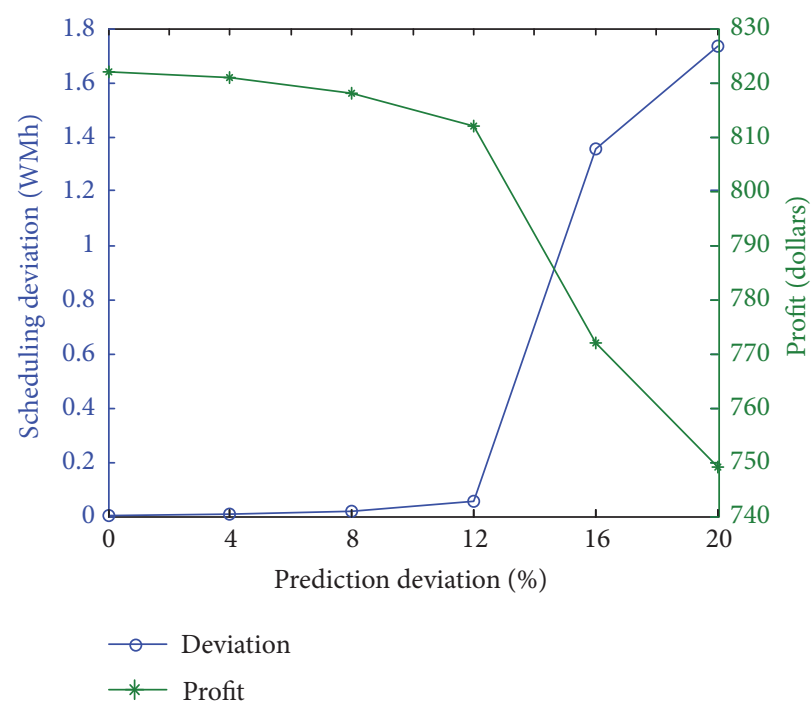

FIGURE 9: Profit and scheduling deviation in winter.

\section{Conclusions}

In this paper, a two-level IDR framework is proposed by introducing load aggregators (i.e., S.E. Hub operators in the paper). Based on the framework, noncooperative game model is modeled to search the optimal bidding strategy for S.E. Hub operators in day-ahead market, and then it is proved that the bidding game has unique Nash equilibrium. While in real-time scheduling, optimization dispatching model is modeled considering the consumption characteristic of each type of household appliances. In the case study, simulation results in summer and winter are presented. The results demonstrate that, by implementing the IDR framework, smart grid, S.E. Hub operators, and residential users all have the benefit. For smart grid, PAR will be improved effectively. For operators and users, they can obtain the economic income. In addition, by analyzing the influence of prediction accuracy on IDR, it is shown that enhancing prediction accuracy of flexible resource will contribute to the profit of load aggregators.

\section{Appendix}

\section{A. Proof of Lemma 1}

Obviously, the function $R_{n}\left(\boldsymbol{L}_{n}, \boldsymbol{L}_{-n}\right)$ is continuously differentiable in $\boldsymbol{L}_{n}$. As for the proof of function $R_{n}\left(\boldsymbol{L}_{n}, \boldsymbol{L}_{-n}\right)$ is concave in over the set $\boldsymbol{L}_{n}$, that is to prove Hessian matrix of $R_{n}\left(\boldsymbol{L}_{n}, \boldsymbol{L}_{-n}\right)$ is negative definite. Accordingly, Hessian matrix of $R_{n}\left(\boldsymbol{L}_{n}, \boldsymbol{L}_{-n}\right)$ is

$$
\nabla_{\mathbf{L}_{n}}^{2} R_{n}\left(\boldsymbol{L}_{n}, \boldsymbol{L}_{-n}\right)=\operatorname{diag}\left[2 \dot{p}_{t}\right]_{t=1}^{T}=\operatorname{diag}\left[2 a_{t}\right]_{t=1}^{T}
$$


Since $a_{t}<0$, (A.1) is a diagonal matrix with all diagonal elements being negative. That is, Hessian matrix of $R_{n}\left(\boldsymbol{L}_{n}, \boldsymbol{L}_{-n}\right)$ is negative definite. Therefore, for each fixed $L_{-n}$, the function $R_{n}\left(\boldsymbol{L}_{n}, \boldsymbol{L}_{-n}\right)$ is concave in over the set $\boldsymbol{L}_{n}$.

\section{B. Proof of Proposition 4}

Based on Lemma 3, if VI problem VI $(\mathscr{L}, \boldsymbol{F})$ only possesses one solution, then Proposition 4 can be proved. According to [26], $\operatorname{VI}(\mathscr{L}, \boldsymbol{F})$ will have a unique solution if $\boldsymbol{F}(\boldsymbol{L})$ is strictly monotone over since the feasible set $\mathscr{L}$ is compact and convex.

In order to prove the strict monotonicity of $\boldsymbol{F}(\boldsymbol{L})$, that is to prove

$$
\sum_{t=1}^{T} \sum_{n=1}^{N}\left[\left(x_{n}^{t}-y_{n}^{t}\right)\left(\nabla_{x_{n}^{t}} R_{n}(\boldsymbol{x})-\nabla_{y_{n}^{t}} R_{n}(\boldsymbol{y})\right)\right]>0
$$

where $\boldsymbol{x}=\left\{\boldsymbol{x}_{n}\right\}_{n=1}^{N} \in \mathscr{L}, \boldsymbol{y}=\left\{\boldsymbol{y}_{n}\right\}_{n=1}^{N} \in \mathscr{L}$.

Let $\boldsymbol{l}^{t}=\left\{x_{1}^{t}, x_{2}^{t}, \cdots, x_{N}^{t}\right\}$ and $\boldsymbol{j}^{t}=\left\{y_{1}^{t}, y_{2}^{t}, \cdots, y_{N}^{t}\right\}$; then (B.1) can be rewritten as

$$
\sum_{t=1}^{T}\left[\left(\boldsymbol{l}^{t}-\boldsymbol{j}^{t}\right)\left(\nabla_{\boldsymbol{l}^{t}} R_{n}^{t}\left(\boldsymbol{l}^{t}\right)-\nabla_{\boldsymbol{j}^{t}} R_{n}^{t}\left(\boldsymbol{j}^{t}\right)\right)\right]>0
$$

where

$$
R_{n}^{t}\left(\boldsymbol{l}^{t}\right)=p_{t}\left(\sum_{n=1}^{N} x_{n}^{t}\right) x_{n}^{t}
$$

and

$$
\nabla_{\boldsymbol{l}^{t}} R_{n}^{t}\left(\boldsymbol{l}^{t}\right)=\left[\nabla_{x_{1}^{t}} R_{n}^{t}\left(\boldsymbol{l}^{t}\right), \nabla_{x_{2}^{t}} R_{n}^{t}\left(\boldsymbol{l}^{t}\right), \cdots \nabla_{x_{N}^{t}} R_{n}^{t}\left(\boldsymbol{l}^{t}\right)\right]^{T}
$$

If the following condition is satisfied, then (B.2) will be held.

$$
\left(\boldsymbol{l}^{t}-\boldsymbol{j}^{t}\right)\left(\boldsymbol{g}_{t}\left(\boldsymbol{l}^{t}\right)-\boldsymbol{g}_{t}\left(\boldsymbol{j}^{t}\right)\right)>0 \quad \forall t \in \boldsymbol{T}
$$

where $\boldsymbol{g}_{t}\left(\boldsymbol{l}^{t}\right)=\nabla_{\boldsymbol{l}^{t}} R_{n}^{t}\left(\boldsymbol{l}^{t}\right)$.

To prove (B.5), that is to prove Jacobian matrix of $\boldsymbol{g}_{t}\left(\boldsymbol{l}^{t}\right)$ is negative definite, assume that $\boldsymbol{G}_{t}\left(\boldsymbol{l}^{t}\right)=\nabla_{\boldsymbol{l}^{t}} \boldsymbol{g}_{t}\left(\boldsymbol{l}^{t}\right)$; then

$$
\boldsymbol{G}_{t}\left(\boldsymbol{l}^{t}\right)+\boldsymbol{G}_{t}\left(\boldsymbol{l}^{t}\right)^{T}=2 a_{t}\left(\mathbf{1 1}^{T}+\mathbf{I}\right)
$$

where I is a unit matrix and $\mathbf{1}$ is an $N \times 1$ matrix where all elements are 1 . Characteristic values of matrix $11^{T}+\mathbf{I}$ are 1 and $N+1$; hence, the characteristic values of $\boldsymbol{G}_{t}\left(\boldsymbol{l}^{t}\right)+\boldsymbol{G}_{t}\left(\boldsymbol{l}^{t}\right)^{T}$ are $2 a_{t}$ and $2(N+1) a_{t}$. Due to $a_{t}<0, \boldsymbol{G}_{t}\left(\boldsymbol{l}^{t}\right)+\boldsymbol{G}_{t}\left(\boldsymbol{l}^{t}\right)^{T}$ is negative definite matrix. That is, $\boldsymbol{F}(\boldsymbol{L})$ is strictly monotone. Therefore, the formulated game has the unique Nash equilibrium.

\section{Nomenclature}

$N$ : $\quad$ Set of smart energy hubs

$T: \quad$ Set of dispatching time slots

M: $\quad$ Set of residential users in S.E. Hub $n$

$A_{m}^{i}$ : $\quad$ Appliance set for type $i$ of load

$\boldsymbol{L}_{n}: \quad$ Bidding strategy set for S.E. Hub operator

$$
n
$$

$\boldsymbol{L}_{-\boldsymbol{n}}: \quad$ Bidding strategy set of other S.E. Hub operators except operator $n$

$t$ : Any dispatching time slot

$L_{n}^{t}: \quad$ Bidding amount in time slot $t$

$L_{n_{i}}^{\mathrm{M} t}$ : $\quad$ Maximal bidding amount of load $i$

$L^{t}$ : Total bidding amount in time slot $t$

$H_{n}^{t}$ : Gas boiler's heat output in S.E. Hub $n$

$\gamma_{n}^{t}: \quad$ Gas consumption rate in S.E. Hub $n$

$p_{t}: \quad$ Bidding price in time slot $t$

$a_{t}, b_{t}$ : Parameters of bidding price

$\gamma_{i}: \quad$ Compensation price for load $i$

$p_{t}^{h}$ : Thermal energy price selling to users

$c_{\text {gas }}$ : Price of natural gas

$\stackrel{g}{t}_{n}^{t}: \quad$ Gas consumption amount in time slot $t$

$\widetilde{L}_{n}^{t}: \quad$ Real-time dispatching amount in S.E. Hub $n$

$a_{m}^{i}: \quad$ Any appliance in the set $A_{m}^{i}$

$P_{t}^{a_{m}^{i}}: \quad$ Consumption power of appliance $a_{m}^{i}$

$Q_{a_{m}^{1}}$ : Daily energy consumption of appliance $a_{m}^{1}$

$P_{t}^{a_{m}^{1}}$ : Consumption power of appliance $a_{m}^{1}$

$Q_{a_{m}^{2}}$ : Daily energy consumption of appliance $a_{m}^{2}$

$P_{t}^{a_{m}^{2}}$ : Consumption power of appliance $a_{m}^{2}$

$\tau_{o n}^{a_{m}^{3}}$ : Shortest turn-on time of appliance $a_{m}^{3}$

$\tau_{o f f}^{a_{m}^{3}}: \quad$ Longest turn-off time of appliance $a_{m}^{3}$

$Q_{a_{m}^{4}}$ : Daily energy consumption of appliance $a_{m}^{4}$

$P_{t}^{a_{m}^{4}}: \quad$ Consumption power of appliance $a_{m}^{4}$

$P_{t}^{S u_{m}}$ : Provided energy of substitute device

$S u_{m}(t)$ : Operation state of substitute device.

\section{Data Availability}

The data used to support the findings of this study are available from the corresponding author upon request.

\section{Conflicts of Interest}

The authors declare that they have no conflicts of interest.

\section{Acknowledgments}

This work is supported in part by National Natural Science Foundation of China (Grant no. 51607036), ADN Comprehensive Demonstration Project of Smart Grid Application Demonstration Area in Suzhou Industrial Park, Yunnan Provincial Talents Training Program (Grant no. KKSY201704027), and Scientific Research Foundation of 
Yunnan Provincial Department of Education (Grant no. 2018JS032).

\section{References}

[1] S. Gyamfi and S. Krumdieck, "Price, environment and security: Exploring multi-modal motivation in voluntary residential peak demand response," Energy Policy, vol. 39, no. 5, pp. 29933004, 2011.

[2] J. Wang, C. N. Bloyd, Z. Hu, and Z. Tan, "Demand response in China," Energy, vol. 35, no. 4, pp. 1592-1597, 2010.

[3] S. Bahrami and A. Sheikhi, "From demand response in smart grid toward integrated demand response in smart energy Hub," IEEE Transactions on Smart Grid, vol. 7, no. 2, pp. 650-658, 2016.

[4] Y. Zhao, K. Peng, B. Xu, H. Li, Y. Liu, and X. Zhang, "Bilevel optimal dispatch strategy for a multi-energy system of industrial parks by considering integrated demand response," Energies, vol. 11, no. 8, p. 1942, 2018.

[5] S. Mohagheghi, F. Yang, and B. Falahati, "Impact of demand response on distribution system reliability," in Proceedings of the 2011 IEEE PES General Meeting: The Electrification of Transportation and The Grid of The Future, pp. 1-7, USA, 2011.

[6] X. Wang and Y. Gong, "Air conditioner fast dispatching model based on load aggregator and direct load control," The Journal of Engineering, vol. 2017, no. 13, pp. 2535-2538, 2017.

[7] X. Liu, B. Gao, and J. Luo, "Non-cooperative game based hierarchical dispatch model of residential loads," Dianli Xitong Zidonghua/Automation of Electric Power Systems, vol. 41, no. 14, pp. 54-60, 2017.

[8] H. Mohsenian-Rad, "Optimal demand bidding for timeshiftable loads," IEEE Transactions on Power Systems, vol. 30, no. 2, pp. 939-951, 2015.

[9] M. Kohansal and H. Mohsenian-Rad, "Price-maker economic bidding in two-settlement pool-based markets: the case of timeshiftable loads," IEEE Transactions on Power Systems, vol. 31, no. 1, pp. 695-705, 2016.

[10] M. C. Caramanis and J. M. Foster, "Uniform and complex bids for demand response and wind generation scheduling in multi-period linked transmission and distribution markets," in Proceedings of 50th IEEE Conference on Decision and Control and European Control, pp. 4340-4347, 2011.

[11] H. Yan and H. Yan, "Optimal energy purchases in deregulated California energy markets," in Proceedings of the IEEE Power Engineering Society Winter Meeting, 2000, pp. 1249-1254, Singapore, January 2000.

[12] C. Zhang, Q. Wang, J. Wang, P. Pinson, J. M. Morales, and J. Ostergaard, "Real-time procurement strategies of a proactive distribution company with aggregator-based demand response," IEEE Transactions on Smart Grid, vol. 3053, no. c, p. 1, 2016.

[13] N. Ruiz, I. Cobelo, and J. Oyarzabal, "A direct load control model for virtual power plant management," IEEE Transactions on Power Systems, vol. 24, no. 2, pp. 959-966, 2009.

[14] S. Rahnama, J. D. Bendtsen, J. Stoustrup, and H. Rasmussen, "Robust aggregator design for industrial thermal energy storages in smart grid," IEEE Transactions on Smart Grid, vol. 8, no. 2, pp. 902-916, 2017.

[15] S. Chen, Q. Chen, and Y. Xu, "Strategic Bidding and Compensation Mechanism for a Load Aggregator With Direct Thermostat Control Capabilities," IEEE Transactions on Smart Grid, vol. 9, no. 3, pp. 2327-2336, 2018.
[16] C. Gao, Q. Li, and Y. Li, "Bi-level optimal dispatch and control strategy for air-conditioning load based on direct load control," in Proceedings of the CSEE, vol. 34, pp. 1546-1555, 2014.

[17] Z. Pan, C. Gao, and S. Liu, "Research on charging and discharging dispatch of electric vehicles based on demand side discharge bidding," Dianwang Jishu/Power System Technology, vol. 40, no. 4, pp. 1140-1146, 2016.

[18] A. Sheikhi, M. Rayati, and A. M. Ranjbar, "Dynamic load management for a residential customer; Reinforcement Learning approach," Sustainable Cities and Society, vol. 24, pp. 42-51, 2016.

[19] A. Sheikhi, M. Rayati, and A. M. Ranjbar, "Demand side management for a residential customer in multi-energy systems," Sustainable Cities and Society, vol. 22, pp. 63-77, 2016.

[20] A. Sheikhi, M. Rayati, S. Bahrami, and A. M. Ranjbar, "Integrated demand side management game in smart energy hubs," IEEE Transactions on Smart Grid, vol. 6, no. 2, pp. 675-683, 2015.

[21] A. Sheikhi, S. Bahrami, and A. M. Ranjbar, "An autonomous demand response program for electricity and natural gas networks in smart energy hubs," Energy, vol. 89, pp. 490-499, 2015.

[22] A. Mohsenian-Rad, V. Wong, J. Jatskevich, R. Schober, and A. LeonGarcia, "Autonomous demand-side management based on game-theoretic energy consumption scheduling for the future smart grid," IEEE Transactions on Smart Grid, vol. 1, no. 3, pp. 320-331, 2010.

[23] X. Liu, S. Wang, and J. Sun, "Energy management for community energy network with CHP based on cooperative game," Energies, vol. 11, no. 5, p. 1066, 2018.

[24] H. M. Soliman and A. Leon-Garcia, "Game-theoretic demandside management with storage devices for the future smart grid," IEEE Transactions on Smart Grid, vol. 5, no. 3, pp. 1475-1485, 2014.

[25] F. Facchinei and J.-S. Pang, Finite-Dimensional Variational Inequalities and Complementarity Problems, vol. I and II, Springer, New York, NY, USA, 2003.

[26] G. Scutari, D. P. Palomar, F. Facchinei, and J.-S. Pang, "Monotone games for cognitive radio systems," in Distributed decision making and control, vol. 417 of Lect. Notes Control Inf. Sci., pp. 83-112, Springer, London, 2012. 


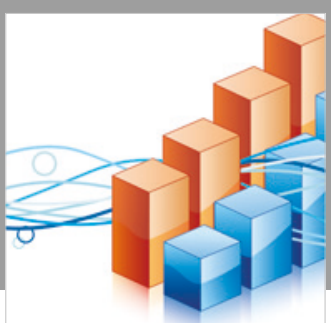

Advances in

Operations Research

\section{-n-m}
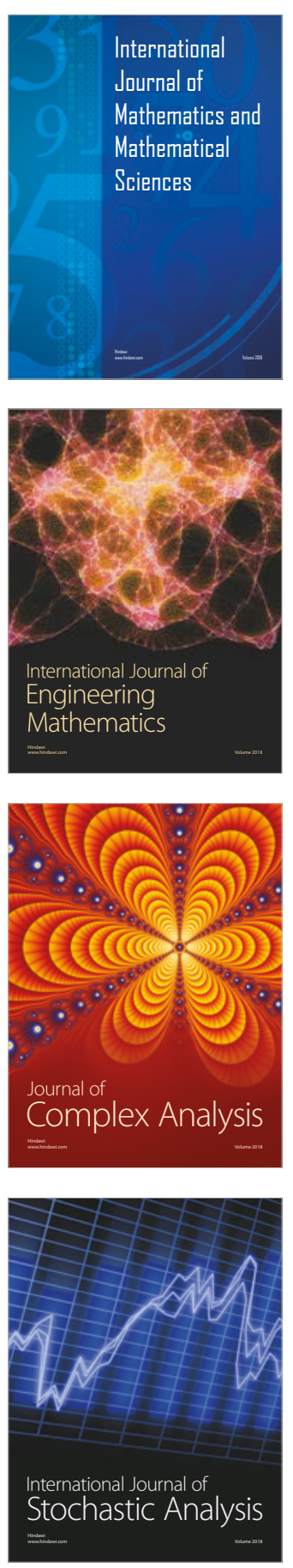
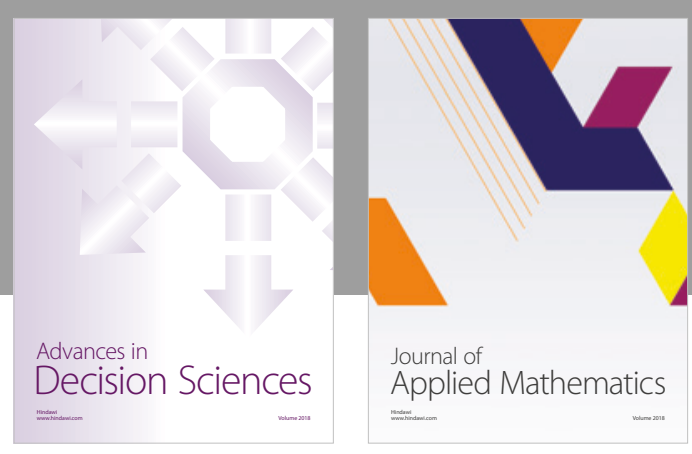

Journal of

Applied Mathematics
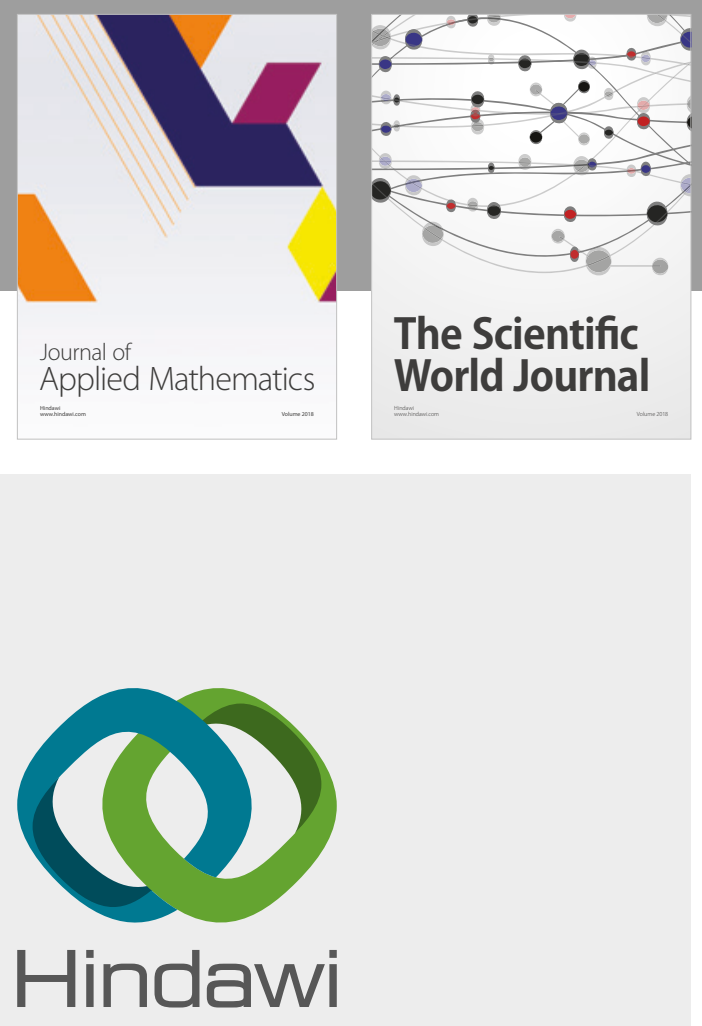

Submit your manuscripts at

www.hindawi.com

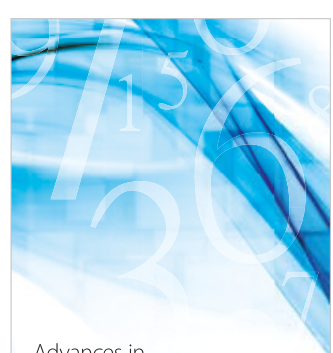

Advances in
Numerical Analysis
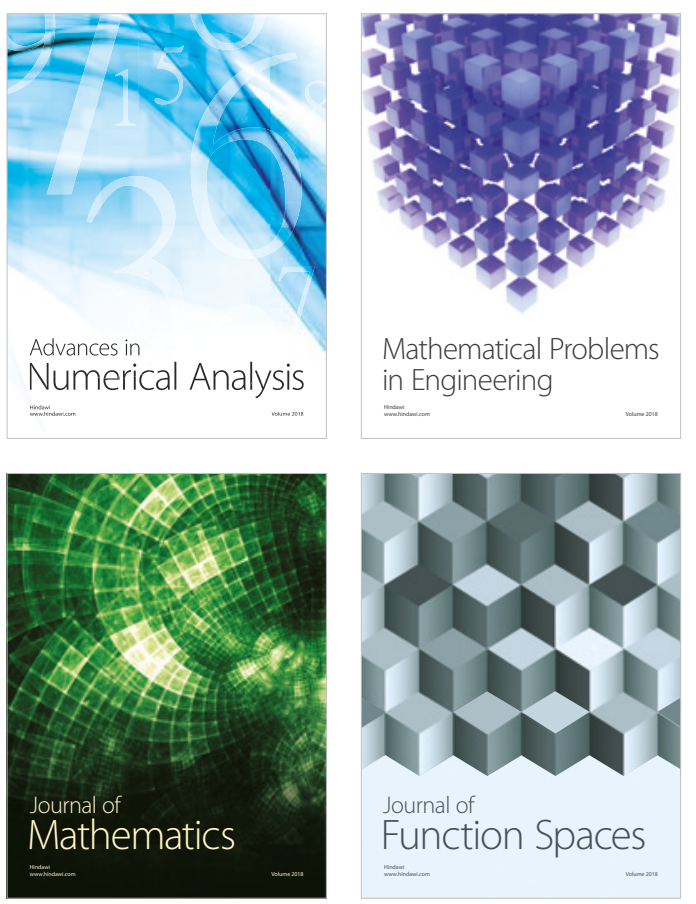

Mathematical Problems in Engineering

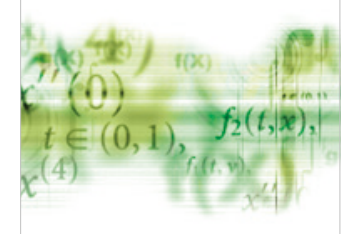

International Journal of

Differential Equations

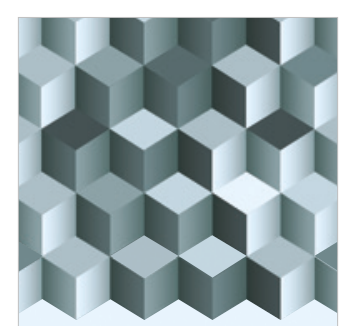

Journal of

Function Spaces

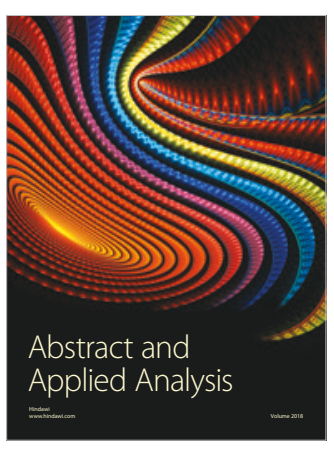

The Scientific

World Journal

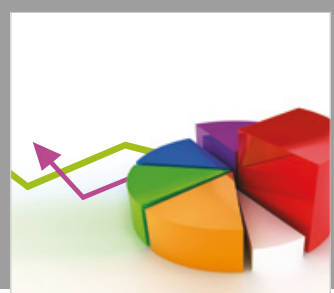

Journal of

Probability and Statistics
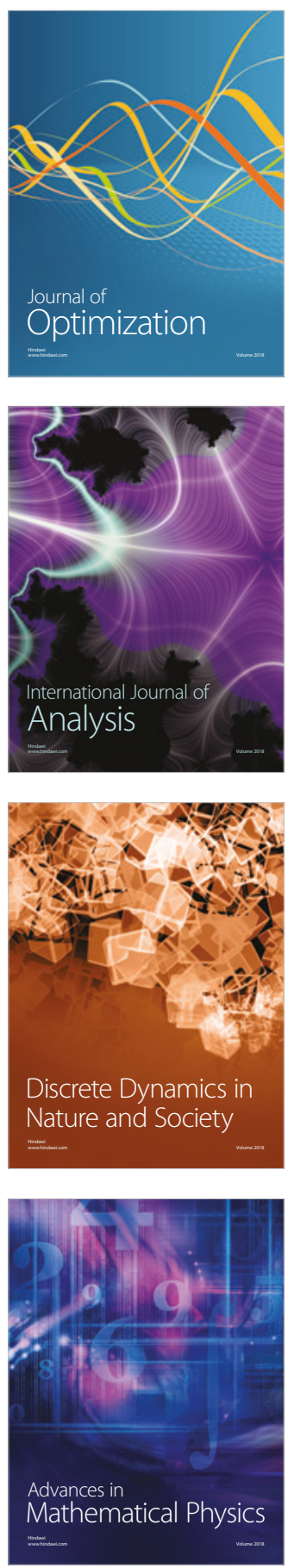